\title{
The Use of High Pressures for Steam Turbine Installations.
}

\author{
By Stanley S. Cook.
}

THREE different methods are available for the measurement of the performance of a steam engine, and serve that purpose from different points of view according to the particular feature of merit it is desired to emphasise. Thus, from the point of view of the manufacturer whose aim is to produce a turbine of the highest efficiency, that is, one which will convert into useful work the highest possible proportion of the pressure energy that is available in the steam, a figure expressing this proportion appears to meet the requirements of the case. This figure is almost (that is, except for the work of restoring the water of condensation to the boiler) identical with the 'efficiency ratio' as defined by Willans and Sankey in terms of the Rankine cycle, a ratio for many years accepted as the criterion of merit of a steam engine. The user of a turbine, on the other hand, is not so interested in what may be called the intrinsic efficiency of the engine, as in knowing how many pounds of steam per hour he has to generate in his boilers in order to produce a horsepower or kilowatt of output; consequently, a more significant expression of the engine's efficiency from his point of view is the consumption in pounds of steam per horse-power hour, under standard conditions of steam generation.

The latter method of statement of performance has also been commonly adopted, is if anything more familiar than the former and in a sense more practical. Used without qualification, however, it gives the manufacturer of the engine credit for any improvement he may make or find in the vacuum or in the steam conditions, although these are frequently taken into account by means of correction factors which reduce the consumption rate to a standard basis.

It must be recognised, however, that neither of these values supplies the user with the exact information he requires as to the cost of producing a unit of energy. It might easily be found, for example, that as the result of utilising higher pressures or lower vacua the efficiency ratio was reduced, but all the same a better overall result secured as measured in pounds of steam per horsepower, and even actually. At the same time, an improvement in consumption rate by improved steam conditions and improved vacua fails to take into account the increased cost of generating the steam under the new conditions.

A third and more comprehensive method is required to give an adequate measure of the performance of the steam engine as a heat engine. It must be remembered that a turbine is not correctly speaking a heat engine, but only a part of it. The expansion which is carried out in the turbine is only one of the processes in the complete cycle of the heat engine, which consists also of processes of heat rejection, re-compression and heat reception. The complete heat engine therefore includes the boiler in which heat is given to the steam, the condenser in which heat is extracted from it, and the condensate pump and feed pump which restore the condensed steam to boiler pressure.

The third method therefore relates to the heat engine as a whole, and aims at expressing its performance as a ratio of the net output, in equivalent heat units, to the heat energy latent in the fuel consumed. Such a ratio is known as the overall thermal efficiency. Its use is clearly necessary if satisfactory comparison is to be made between heat engines of different types, employing thermodynamic cycles of different character.

Inquiry must therefore be made into the conditions which make for the highest thermal efficiency. Now if it be presupposed that the highest possible efficiency of heat transmission in the boiler has been obtained, and the highest possible intrinsic efficiency of the turbine, there still remains another factor of supreme importance, namely, the efficiency of the thermodynamic steam cycle which alone determines the ratio between the heat supplied to the steam and the pressure energy that is available for the turbine. The turbine is able to use efficiently all the available energy that can be given to it, and it therefore remains to provide that the efficiency of the thermodynamic cycle shall be made as high as possible subject to practical conditions. At this point the problem becomes a thermodynamic one, and this ratio is a function of the conditions of heat reception and heat rejection.

The law which governs this question was enunciated by Carnot more than a century ago. It is that in order to obtain the maximum thermodynamic efficiency the temperature of heat reception must be made as high as possible and the temperature of heat rejection as low as possible. In the perfect Carnot cycle heat is received at a constant high temperature and abstracted at a constant low temperature. The heat received is proportional to the absolute temperature of reception, and the heat abstracted to the absolute temperature of rejection. The difference between these quantities of heat is the amount transformed into work, so that the thermodynamic efficiency of the perfect engine becomes $\left(T_{1}-T_{2}\right) / T_{1}$ where $T_{1}$ and $T_{2}$ are the higher and lower absolute temperatures. It is easily seen that this ratio is increased by increasing $\mathrm{T}_{1}$, or by decreasing $\mathrm{T}_{2}$, or both.

The past success of the steam engine as a prime mover is due to two causes, the high value of the latent heat of steam and its ability to work on what is known as the Rankine Cycle. In the latter the compression stage of the Carnot cycle is replaced by the simple process of elevating the condensed water to boiler pressure. The consequent reduction of negative work gives this cycle a great practical superiority.

On the other hand, the employment of such a cycle involves a departure from the above-mentioned principle of heat reception at a constant maximum temperature. The heat is in fact supplied to the steam at a variety of temperatures. The feed water has to be raised from condenser temperature to the boiling point corresponding to the pressure of the boiler. Evaporation in the boiler takes place at that temperature, and the temperature of the steam is then raised continuously to the maximum temperature of superheat. The thermodynamic efficiency of each portion of the heat so supplied is conditioned by the ratio of the absolute temperature of supply to the absolute temperature at which all the heat is abstracted in the condenser. 
Now the heat given to the steam during the process of evaporation, namely, the latent heat, is a large proportion of the total heat supplied. For example, with a boiler pressure of $250 \mathrm{lb}$. per square inch, a superheat of $200^{\circ} \mathrm{F}$. and a condenser vacuum of $29 \mathrm{in}$. $\mathrm{Hg}$. the latent heat is $836 \mathrm{~B}$. Th. U. per lb. of steam out of a total supplied of $1276 \mathrm{~B}$. Th. U. per $\mathrm{lb}$. during the three processes just mentioned, or 65.5 per cent of that total. Omitting the heat required to heat the feed water, since, as will be seen presently, the disability of the low temperature of this process can be overcome, the total heat required for evaporation and superheat is $95 \circ \mathrm{B}$. Th. U., of which the latent heat added during evaporation is 88 per cent. It is clearly, therefore, of importance from the point of view of obtaining the highest thermodynamic efficiency of the cycle that the heat supplied during the stage of evaporation shall be supplied at as high a temperature as possible. The temperature of this part of the heat reception can only be raised by increasing the boiler pressure.

At the same time, an increase of the maximum temperature of the superheated steam will lead to further improvement in efficiency, since this means additional heat reception at the highest temperature. In the case of superheat, there is the additional advantage that with a higher degree of initial superheat, the steam is in a dryer condition in the final stages of its expansion.

The circumstances are best visualised by means of an entropy temperature diagram. Fig. I is such a

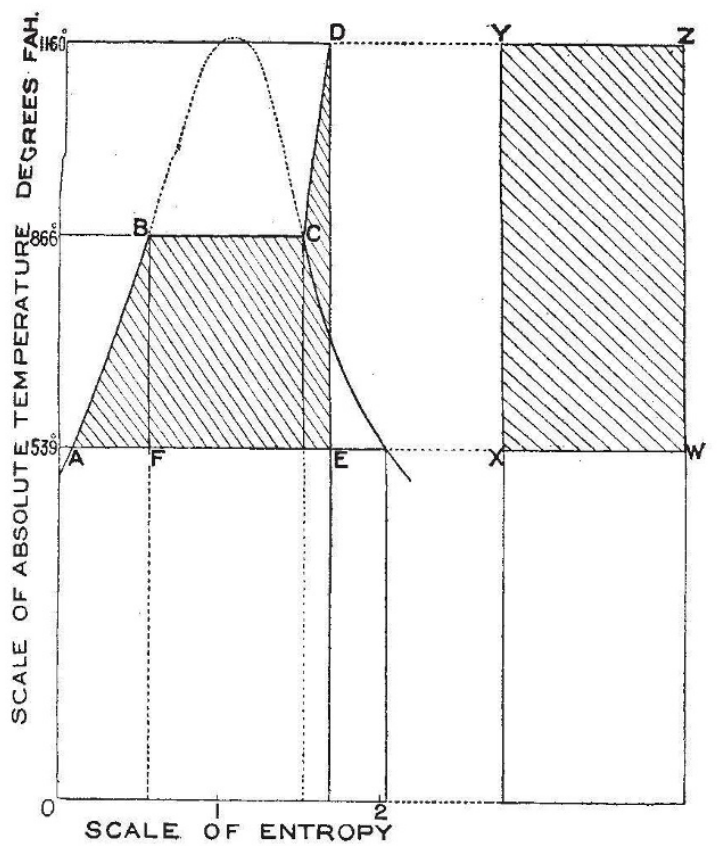

FIG. r.-Entropy temperature diagram for the Rankine cycle for steam with boiler pressure $25^{\circ} \mathrm{lb}$., condenser temperature $79^{\circ} \mathrm{F}$. and superheat to $700^{\circ} \mathrm{F}$.

diagram representing the Rankine cycle for steam with a boiler pressure of $250 \mathrm{lb}$. gauge, a condenser temperature of $79^{\circ} \mathrm{F}$., and superheat up to a maximum temperature of $700^{\circ} \mathrm{F}$. Each stage of the cycle is represented by a curve on the diagram, and the property of the diagram is that, with absolute temperature as the vertical ordinate, the area vertically beneath any curve, or portion of a curve, which corresponds to a change of state is proportional to the heat taken in or given out during that change. The rising curve $A B$ represents the heating of the feed water to boiler temperature, by whatever means it is carried out ; the line $B C$ represents the generation of steam and is horizontal because this takes place at constant temperature; the rising curve $C D$ represents the superheating of the steam. No heat is taken in or given out during expansion in the turbine, so that the vertical line $D E$ represents expansion of the steam down to the temperature of the condenser. In the condenser, condensation takes place at constant temperature, and is therefore represented by a horizontal line $E A$, which completes the cycle. The area enclosed by the cycle $A B C D E A$ represents the excess of the heat taken in from the boiler along $A B C D$, over that rejected into the condenser along $E A$. This area therefore represents the energy which is available for conversion into work.

The shortcomings of this cycle can be seen at a glance if we compare it with another cycle $X Y Z W X$ in which all the heat received from the source is taken in at the highest temperature along $Y Z$, and that rejected is rejected at the lowest temperature along $W X$. Here the work done is represented by the area of the rectangle $X Y Z W$, whilst the heat taken in is represented by the larger rectangle from $Y Z$ down to the base line. The proportion of the work done to the heat taken in is much higher than in the cycle $A B C D E$, and this is clearly seen to be due to the high temperature at which all the heat is taken in along $Y Z$. The underlying areas of the first diagram shew us at once that, of the heat taken in along $A B$, that is, during the heating of the cold feed in the boiler, only a small percentage is converted into useful work. The heat taken in along $B C$, which is the latent heat of the steam, contributes a larger percentage, but still considerably less than the maximum. Even that received along $C D$ during the process of superheating, yields less than the ideal maximum efficiency.

While the diagram thus exhibits the shortcomings of the Rankine cycle in comparison with an ideal Carnot cycle, it is of interest to remark that similar departures from the ideal are present in any type of engine in practical use. The internal-combustion engine, for example, contains an even greater departure from the ideal of constant temperature reception of heat, although its actual temperatures both of reception and rejection are higher throughout than in the steam engine.

Returning to the Rankine cycle for steam, it has been stated that the heat received during evaporation is a large proportion of the total heat received. That this is the case is readily seen from the diagram, this heat being represented by the area below $B C$ down to the base line. By what is known as cascade feed heating, that is by heating the feed water by steam bled from the turbine at suitable successive stages, it is possible to a large extent to eliminate that part of the diagram which underlies $A B$, since by this process if carried out in its entirety the heat represented by the area from $A B$ down to the base line can be saved, at the expense of a loss of work which can be shown to be equivalent to the area $A B F$ for each lb. of steam. To raise the temperature of reception of the heat absorbed during

$$
\text { NO. 2969, VOL. I I } 8 \text { ] }
$$


evaporation, which in the case already considered is 88 per cent. of the total heat absorbed along $B C D$, would clearly result in considerable improvement of the efficiency of the thermodynamic cycle. Thus not only high temperatures, as in the case of the adoption of high superheat, but also high boiler pressures, lead to a substantial increase in over-all thermal efficiency.

The question of the improvement of the thermal efficiency of the steam engine by such means is one of prominent interest in the engineering world at the present time. Advances in this direction have already been made for land installations, and recently we have witnessed an application of the same principles to marine work, which had been awaited with keen interest, and was not without an historic setting. It is just a quarter of a century since the first commercial application of the steam turbine to marine work was made in the Clyde river steamer the King Edward, which, built in I90r, has continued in successful service season by season up to the present time. A new vessel, which has a high-pressure steam installation of geared turbines on the lines indicated above, has been built for the same owners and for the same service. This vessel, which has been named King George $V$, becomes in its turn the pioneer of the new system.

\section{Malaria and the Mosquito.}

THE enthusiast who is 'eradicating' malaria by any or all of the well known methods, should cease for a while from his labours and study the short report recently issued by the League of Nations and referred to below. 1 In Col. James's words, "He will at least realise what a great waste of effort is involved in measures directed against the breeding-places of mosquitoes as a whole and even in similar measures directed against one species. He will begin to appreciate how the secret of a successful control of malaria lies not in the general knowledge that the disease is spread by mosquitoes of a certain kind, but in the particular exact knowledge of the life history of the few individual mosquitoes which succeed in becoming transmitters of the disease."

This is a refreshingly new aspect of the problem. The enthusiast eradicates all mosquitoes. He does not stop to distinguish a Culicine (non-malaria) from an Anopheline (malaria) mosquito; he is out to destroy them all. Now he is advised to study not only the species of Anopheles which are concerned in transmission, for all are not, but also actually to concern himself about infected individuals ! Why should he do this? It is because "Malaria is essentially a household disease and particularly a disease of certain kinds of houses .... Malaria should be dealt with in the houses of the people rather than in the environment." We have called this a new aspect of the problem, but the author points out that some twenty-five years ago Stephens and Christophers in their reports to the Royal Society directed attention to "fever houses." They state: "We may look upon such a house and its accessory hovels as one infected with malaria or as 'a fever house.' Such is the universal condition of European houses indeed in the remote stations situated in the African bush. It is in such houses that the malarial cachectic is living, exposed to frequent or even constant re-infection and in which every traveller staying the night is liable to infection. From such fever houses the majority of our cases of blackwater have come."

It has always been somewhat disconcerting that while, for example, in a native African village more than 75 per cent. of the children may be infected with malaria, yet the infection rate of sporozoites (infective stage of the malaria parasite) among the anophelines may be low, less than 5 per cent. Once infected, however, a single mosquito becomes

${ }^{1}$ League of Nations: Health Organisation--Malaria Commission. Report on the First Results of Laboratory Work on Malaria in England. By Lieut. Colonel S. P. James and P. G. Shute. (C.H./Malaria/57) (r).) Pp. 30 Geneva : League of Nations; London: Constable and Co., Ltd., 1926.) exceedingly dangerous; how much so is shown by the fact that a mosquito which has lived (in captivity) I-3 months and has bitten nearly half a hundred people in that time may still have numerous sporozoites in its salivary glands and is consequently still potent for mischief. But, on the other hand, if not bitten oneself, one may draw comfort from the fact that a mosquito is gradually purging itself of infection by biting other people, so that while 85 per cent. of mosquitoes showed a salivary glands infection on the 5 oth day, on the 7oth day this was reduced to 20 per cent. (the glands, moreover, containing but few sporozoites), the mosquitoes having bitten about a dozen people in the interval. This at first sight appears inconsistent with the previous statement, but the difference lies in the fact that in the first case the mosquitoes' stock of sporozoites in the glands was being replenished by the oocyst stage present in the stomach, as shown by dissection, while they were absent in the second case.

Col. James's observations lead him to formulate some very important conclusions, namely, that " in Nature the only mosquitoes which succeed in transmitting malaria are those rare individuals who happen to pass their lives in conditions which resemble very closely those which we have found to be essential for the successful transmission of the disease in experimental work" and of these " not many will ultimately become infective." Let the sanitarian then pause and study the individual infective mosquito in the fever house. Here is the real centre of gravity.

Among interesting data with regard to the life of the malaria parasite in the mosquito are those concerning the persistence of oocysts and sporozoites, the young and the mature stages of the malaria parasite in the stomach and salivary glands respectively of captive hibernating mosquitoes, a persistence which is held to explain primary attacks of simple tertian malaria in Nature in the early spring in northern latitudes.

The report concludes with a study of the infected cases from the clinical and microscopical sides. They suggest that we shall have to revise somewhat our idea of the Golgi cycle in relation to the temperature chart, but in blood work we must never forget that the peripheral blood is almost certainly not the same thing as that of the spleen or marrows. Very prudently, we consider, the author refuses to sail on the troubled sea of immunity where shipwrecks are inevitable. The report is written in an agreeably clear fashion with an absence of assumption of authority quite charming. J.W.W.S. 